\title{
Steady Vortex Flows Obtained from a Nonlinear Eigenvalue Problem
}

\author{
Alan Elcrat, Bengt Fornberg, Kenneth Miller \\ Wichita State University, Wichita, KS \\ University of Colorado, Boulder, CO \\ Wichita State University, Wichita, KS
}

\begin{abstract}
A general iterative method for obtaining steady-state two dimensional flows with vorticity is described, and convergence results are given in the case of a bounded domain. We also give computational results for symmetric flow past a circle in the unbounded plane.
\end{abstract}

\section{Introduction}

We study here the classic problem of two dimensional steady-state inviscid flow past a bluff body. We consider flows in which there is a single vortex in the sense that the stream function of the flow is given by the solution of an equation

$$
\triangle \psi=\omega f(\psi)
$$

where $f$ is a nonnegative decreasing function and the positive constant $\omega$ is a vortex strength parameter.

Three types of vortex regions occur. These are "attached" vortices in which the vortex region is connected to the boundary, "isolated" vortices in which the vortex region stands away from the boundary, and "strips" of vorticity extending to infinity along the streamline of symmetry. These three types of solutions will correspond to different choices of the value of the stream function on the boundary of the vortex. This parameter is prescribed in the partial differential equation satisfied by the stream function. The boundary of the vortex is found by observing where the stream function assumes this value.

We have given theoretical results for a bounded domain and a general (possibly discontinuous) bounded decreasing function. A monotone iteration is shown to converge to a solution. The iterants can be described in terms of level sets of approximate stream functions. These reduce to the vortex region in the case of a vortex patch. Results are obtained for each of the three types of solutions, and isolated vortices are shown to be connected with attached vortices with the same circulation by a continuation process.

For related computational work we have restricted attention to symmetric flow past a circular cylinder. Since the flow domain is unbounded the results of our theoretical investigation do not apply directly. In fact in our numerical work we have found it necessary to hold the area of vortices fixed and use non-monotone iterations.

In Section 2 we will summarize our theoretical work. In Section 3 the numerical methods used will be described and a summary of results for different types of solutions will be given. 


\section{Flows in a bounded domain}

Let $\Omega$ be a bounded domain in the plane with boundary $\Gamma$ and $f$ a bounded, non-negative, decreasing (non-increasing) function. The function $f$ may be discontinuous, but we assume that the measure $d f$ has compact support contained in the interval $[\alpha, \beta]$. We prescribe non-negative boundary values $\phi$ for $\psi$ on $\Gamma$, and let $\psi_{0}$ be the harmonic function with these boundary values. The key to the method that we use is in giving a start to iterations which proceed with successive approximations, $\Delta \psi_{n}=\omega f\left(\psi_{n-1}\right)$, for $n>1$. We give as initialization

$$
\psi_{1}(x)=\psi_{0}(x)-\omega \int_{[\alpha, \beta]} \Phi\left(D_{0}(s)\right)(x) d f(s)
$$

where

$$
\Phi(D)(x)=\int_{D} g(x, y) d y
$$

$g$ is the Green's function for $-\Delta$ on $\Omega$, the integral is the Lebesgue-Stieltjes integral, and $D_{0}(s)$, $\alpha \leq s \leq \beta$, is a nondecreasing family of subsets of $\Omega$. The sets $D_{0}(s)$ are an initial guess for the level sets of the vorticity, and the solutions obtained depend very much on this initial guess. For $n \geq 1$ we define $D_{n}(s)=\left\{x \in \Omega: \psi_{n}(x)<s\right\}$. The basic fact is that the condition

$$
D_{0}(s) \subseteq D_{1}(s) \text { for all } s \in[\alpha, \beta]
$$

implies that the sequence $\psi_{n}$ converges to a solution $\psi$ of (1). More precisely, we have

Theorem 1. Assume that $f$ is continuous from the left at $\alpha=\inf \operatorname{supp}(d f)$ and that condition (2) is satisfied. Then the sequence $\psi_{n}$ converges to a solution $\psi$ of $(1), \psi \in C^{1,1}(\Omega) \cap C^{0}(\bar{\Omega})$.

Using this result we can show

Theorem 2. If (2) holds for $\widehat{\omega}$, then for $\omega>\widehat{\omega}$, there is a solution obtained from $D_{0}(s)=\{x \in \Omega$ : $\widehat{\psi}(x)<s\}$ which satisfies $\psi \geq \widehat{\psi}$.

Theorem 3. If $\left\{D_{0}(s): \alpha \leq s \leq \beta\right\}$ is a family of sets satisfying the hypotheses of Theorem 1, $\left|D_{0}(\alpha)\right|>0,\left|\partial D_{0}(\alpha)\right|=0$ and $\operatorname{cl}\left(D_{0}(\alpha)\right) \cap \Gamma=\emptyset$. Then condition (2) is satisfied for all $\lambda$ sufficiently large.

Theorem 3 says that for initial guesses bounded away from the boundary there is always a solution for large $\omega$.

The three types of solutions mentioned in the introduction correspond to different types of initial guesses. In particular consider a boundary function $\phi$ which corresponds to flow through a channel with a bump on the lower channel wall, as shown in Figure 1. The first type of flow is obtained using $D_{0}(s)=\left\{x \in \Omega: \psi_{0}(x)<s\right\}$. The second arise from Theorem 3. The attached vortices can be obtained using

Theorem 4. Suppose $\beta=i n f_{\Gamma} \phi$ and that there is a neighborhood $N$ of $\Gamma_{0}=\Gamma \cap \operatorname{cl}\left(D_{0}(\beta)\right)$ with $\phi \equiv \beta$ on $\Gamma \cap N$. If there exists $k>0$ such that $k \operatorname{dist}\left(D_{0}(t), \Gamma\right) \geq(\beta-t)$ for all $t \in[\alpha, \beta]$, then condition (2) is satisfied for all $\lambda$ sufficiently large.

We note that the special case $\alpha=\beta$ corresponds to a vortex patch, $D_{0}$ is a single set, and the inequality in Theorem 4 is automatically satisfied. In this case we can use results of Turkington 

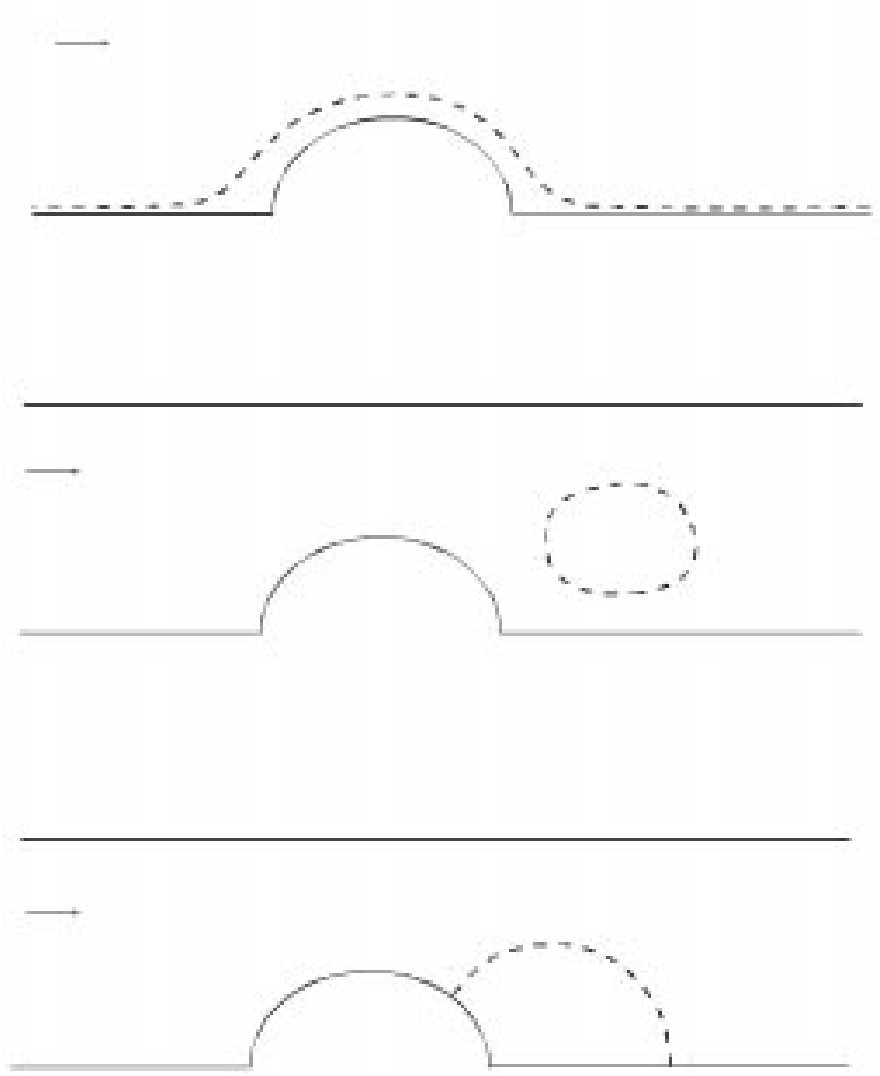

Figure 1: Three types of vortex regions in a channel

[2] to show that small support patch solutions in a neighborhood of a stable point vortex can be continued to attached vortices.

We remark here that analogous results hold for axisymmetric flows.

\section{Computed Solutions}

We have computed solutions for a related problem. The domain is the exterior of a circular cylinder, and we restrict our attention to symmetric flow so that only the upper half plane need be considered. The simple geometry chosen eliminates the need to map out geometric dependencies and the set of solutions is complicated enough to justify this. Also the transformation $w=i \ln (z)$, $z=x+i y$, maps the flow domain to a semi-infinite strip, simplifying discretization.

For the computations we consider only the case where $f=1-H, H$ the Heaviside function, i.e. we are considering only patch type solutions. Therefore $\alpha=\beta$. The parameter $\alpha$ is the value of $\psi$ on the boundary of the vortex. The boundary condition on $\psi$ is $\psi=\psi_{0}, \psi_{0}$ the stream function for potential flow, on the boundary (where $\psi_{0}=0$ ) and at infinity. We obtain vortices attached to the boundary when $\alpha=0$, isolated vortices when $\alpha<0$, and strips of vorticity along the entire boundary when $\alpha>0$.

The theorems described in Section 2 use boundedness of the flow domain in an essential way. 


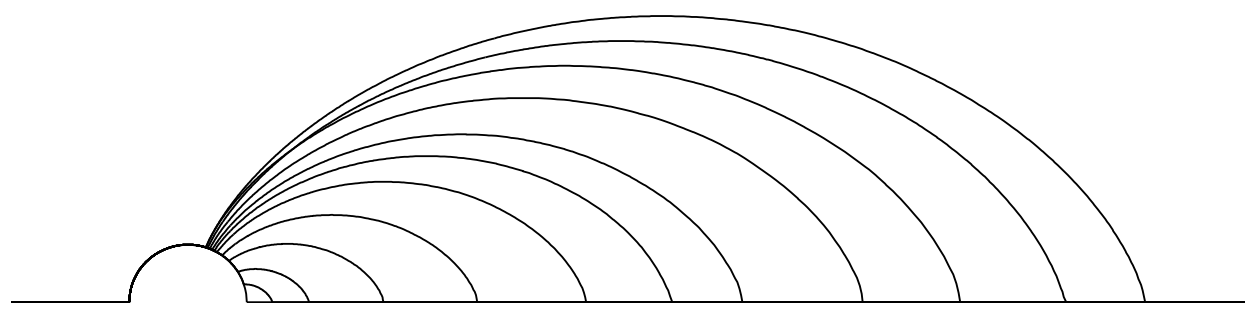

Figure 2: Attached $(\alpha=0)$ trailing vortices, $A$ varying between .1 and 60 .

For this unbounded domain we know of no mathematical work guaranteeing the existence of solutions. Furthermore, implementing the monotone iterations described above proved troublesome and delicate: the iterated vortex regions either filled the computational domain or disappeared. We have instead used iterations in which $\omega$ is allowed to vary and the area of the vortex region held fixed.

The numerical scheme can be summarized as follows:

A) Specify $\alpha$, the desired vortex area $A$, an error tolerance $\epsilon_{A}$, and make an initial guess for the vortex region.

B) Find (by bisection or the secant method) the vorticity level $\omega$ and the corresponding solution $\psi_{n+1}$ to

$$
\Delta \psi_{n+1}=\omega_{n} f\left(\psi_{n}-\alpha\right)
$$

so that

$$
A=\left|\left\{\psi_{n+1}<\alpha\right\}\right|,
$$

is satisfied with tolerance $\epsilon_{A}$.

C) If the new vortex region $\left\{\psi_{n+1}<\alpha\right\}$ differs from the previous vortex region at any grid point, return to step B.

We briefly describe how this scheme is implemented. After transforming to the $w$-plane, $w=i \ln (z)$, (3) becomes a Poisson equation on a semi-infinite strip. To solve the resulting equation we use the standard five point stencil with a uniform mesh in the $w$-plane. To obtain a finite problem we truncate the strip at a finite height, and apply at the top a Robin-type numerical boundary condition which is exact for decaying solutions of the discrete Laplacian on the strip. At each iteration step the differential equation (3) is solved using a fast Poisson solver. The area of the set $\left\{\psi_{n+1}<\alpha\right\}$ is calculated by linear interpolation on the sides of a mesh square.

For attached vortices $(\alpha=0)$ we have used area $=A$ as a continuation parameter. Figure 2 shows the vortex cores for several values of $A$ between 0.1 and 60 . A streamline plot for one of the flows is shown in Figure 3.

These attached flows have a limiting nature for large $A$. First, the attachment point approaches the top of the circle, as suggested in Figure 2. In addition the aspect ratio $\frac{x_{\max }}{y_{\max }}$, where $x_{\max }$ is the distance from the origin to the downstream reattachment point, is roughly 3.3 for large areas. This suggests that the vortex regions approach a Sadovskii vortex (with no vortex sheet) as $a$ increases. A Sadovskii flow ([3]) is the corresponding flow in the full half-plane. The aspect ratio for the Sadovskii vortex has been computed to be 3.338, [4]. 


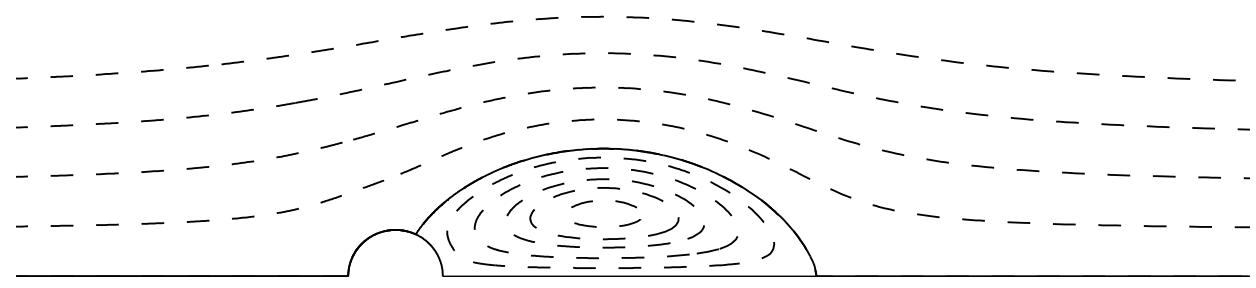

Figure 3: Streamline plot for the attached trailing vortex with $A=18$.

The partial differential equation problem that is being solved can be expected to have more than one solution. In addition to the attached vortices shown in Figure 2 there are also symmetric attached vortices, again parametrized by $A$ for all positive values of $A$. For large $A$ the boundary is convex and the aspect ratio may be expected to asymptotically approach the same limit as above for area tending to infinity. As $A$ is decreased a dimple forms, and eventually (at $A \approx 15$ ) the vortex separates into two symmetric parts as shown in Figure 4.

Before describing the isolated vortices it is helpful to note the possible stationary locations for a point vortex. The complex potential for flow past a semi-circular bump of radius $a$ in the upper half plane with a single point vortex located at $z_{0}$ and circulation $\kappa$ is given by

$$
w=U\left(z+\frac{a^{2}}{z}\right)+i \frac{\kappa}{2 \pi} \log \left(\frac{z-z_{0}}{z-\bar{z}_{0}} \frac{z-a^{2} / z_{0}}{z-a^{2} / \bar{z}_{0}}\right),
$$

where $U$ is the fluid speed at infinity. The condition for stationarity is

$$
\left.\frac{d}{d z}\left[w-\frac{i \kappa}{2 \pi} \log \left(z-z_{0}\right)\right]\right|_{z=z_{0}}=0 .
$$

One set of solutions $z_{0}, \kappa$ is given by points on $r^{2}-a^{2}=2 r y$ with circulation $\kappa=4 \pi U y\left(1-a^{4} / r^{4}\right)$. These are the well-known Föppl vortex locations, shown in column 1, row 1 of Figure 5.

A second family of stationary point vortices, shown in column 1, row 2 of Figure 5 can be obtained by setting $z_{0}=b i, b>a$ in the above equation. The equation obtained reduces to

$$
U\left(1+\frac{a^{2}}{b^{2}}\right)=\frac{\kappa}{2 \pi}\left(\frac{1}{2 b}+\frac{2 a^{2} b}{b^{4}-a^{4}}\right)
$$

For any $b>a$ we can choose $\kappa$ by this equation to obtain a solution. To understand the significance of this solution we can set $a=0$, and observe that we obtain a vortex translating with speed $U$ and circulations $4 \pi b U$, another well known set of point vortices.

In addition to these two families of single stationary point vortex locations, we note (see [1]) that for small $\kappa$ there is a symmetric pair of stationary point vortices with one vortex near each of the

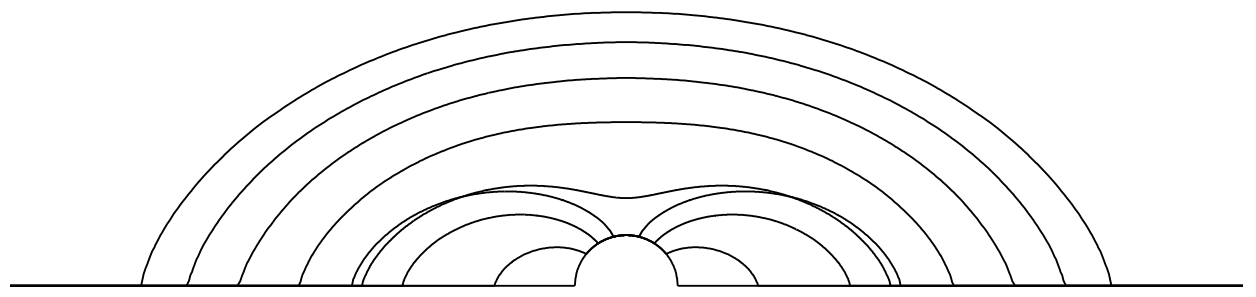

Figure 4: Attached symmetric vortices, $A$ varying between 2 and 75. 


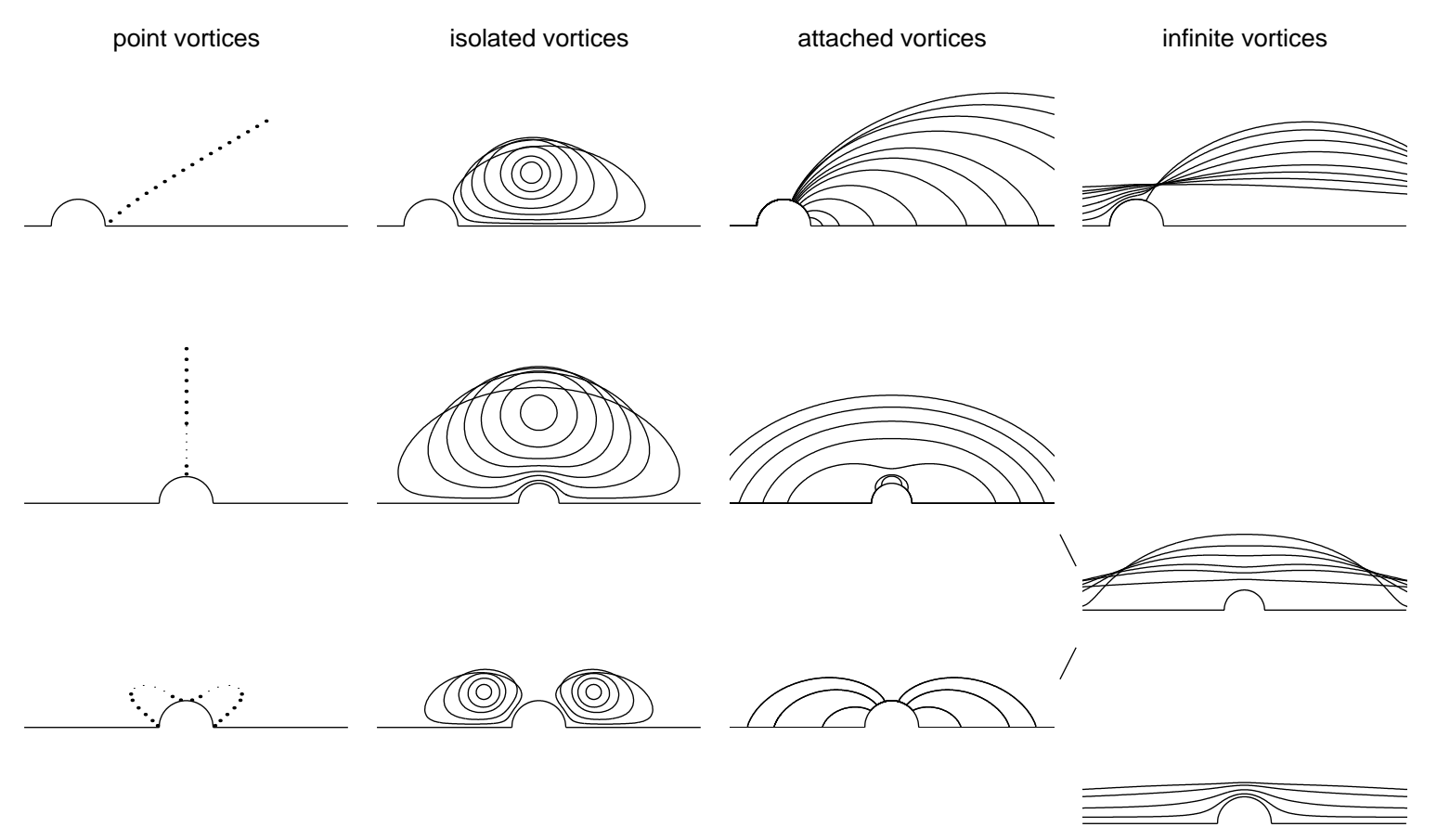

Figure 5: Interconnections between point vortices and the three types of vortex regions that occur.

corners of the intersection of the circle and the x-axis. The locus of such symmetric pairs can be continued to obtain the locus shown in the column 1, row 3 of Figure 5.

For each of these stationary point vortex locations, either single vortex or symmetric pair, there is a family of isolated vortices with the same total circulation $\kappa=\omega A$ as the point vortex. To obtain each member of these families numerically we use a modification of the algorithm described above: we fix $\omega$ and let $\alpha<0$ vary in the inner iteration in order to keep $A$ fixed. By then increasing $A$ incrementally with $\kappa=\omega A$ fixed, we may obtain a family of fixed circulation vortices. Thus we have obtained three two-parameter families of isolated vortices, each parametrized by $\kappa$ and $\alpha$ : trailing vortices desingularizing the Föppl point vortices, connected symmetric vortices desingularizing point vortices on the $y$ axis, and vortices consisting of two components which desingularize the symmetric vortex pairs. The second column of Figure 5 shows three families of isolated vortices with $\kappa$ fixed in each case. In most cases (for those point vortices in the ranges indicated by larger dots in the first column of Figure 5) the resulting family of fixed circulation isolated vortices can be continued to one of the attached vortices $(\alpha=0)$ shown in the third column of Figure 5.

We have also obtained vortices with support extending to infinity. These are obtained as solutions with $\alpha>0$. Since the vortices are unbounded neither area nor circulation is defined. For want of a better choice, we have chosen area inside the computational domain as a computational parameter. Our basic algorithm finds $\omega$ as a function of $A$, but we wanted to fix the physical parameter $\omega$. This was accomplished by making the algorithm into a Fortran function and using an equation solving routine.

For fixed $\omega$ we have found two families of symmetric solutions for positive $\alpha$. For the first of these families the limiting flow as $\alpha \rightarrow 0$ is the flow with no vortex, i.e. potential flow. These are shown at the bottom of the fourth column of Figure 5. For the second symmetric family, the $\alpha=0$ 
solution is the symmetric attached vortex with the same $\omega$. We can also find a family continued from an attached vortex behind the cylinder, as shown in the top of the fourth column in Figure 5. For all three families the continuation cannot be carried out past a critical value of $\alpha$ which depends on $\omega$, but is the same for all three families. For all the solutions shown in the fourth column of Figure 5 the value of $\omega$ is 1 . Note that the three families approach the same solution as $\alpha$ approaches its maximal value. Thus the set of all solutions appears to be connected in some sense.

\section{References}

1 Miller, K. G.: Stationary corner vortex configurations, Z. angew. Math. Phys., 47 (1996), 39-56.

2 SAdovskir, V. S. Vortex regions in a potential stream with a jump of Bernoulli's constant at the boundary. Appl. Math. Mech. 35, 729.

3 Turkington, B. : On steady vortex flow in two dimensions, I, Comm. Partial Differential Equations, 8 (1983), 999-1030.

$4 \mathrm{Wu}$, H. M., Overman, E. A., Zabusky, N.: Steady-state solutions of the Euler equations in two dimensions: rotating and translating $V$-states with limiting cases. I. Numerical algorithms and results, J. Comput. Phys. 53 (1984), 42-71. 